\title{
Sächsischer Datenschutzbeauftragter unterstützt die Datenschutzbehörde von Bosnien-Herzegowina
}

Der Sächsische Datenschutzbeauftragte unterstützt die bosnisch-herzegowinische Datenschutzbehörde in Sarajewo im Rahmen eines EU-Twinning-Light-Projekts vom 1.10.2009 bis zum 31.3.2010.

Die Auftaktveranstaltung am 14.10.2009 im Parlamentsgebäude von Bosnien-Herzegowina wurde durch den Ministerpräsidenten Nikola Spirić eröffnet. Er betonte ebenso wie der Deutsche Botschafter Joachim Schmidt und der Head of Operations der EU-Delegation in Bosnien-Herzegowina Boris Iarotchevitch die Bedeutung des Datenschutzes für das freie und rechtsstaatliche Gemeinwesen.

Um der Europäischen Union beitreten zu können, müssen die Beitrittsstaaten den acquis communautaire der Europäischen Union übernehmen und moderne, effiziente Verwaltungen aufbauen, die in der Lage sind, die Gesetze und Verordnungen in demselben Maße umzusetzen wie die jetzigen Mitgliedsstaaten.

Das im Jahre 1998 im Rahmen der Heranführungsstrategie aufgelegte Twinning-Instrument im Rahmen des Gemeinschaftshilfeprogramms für die Länder Mittel- und Osteuropas (PHARE) verfolgt diese Zielrichtung. Twinning-Projekte dauern 12 bis 24 Monate und werden durch die EU-Kommission mit einem Fiche ausgeschrieben. Dabei wird es zunehmend üblich, dass ein Mitgliedsstaat (Leading Member state) durch andere (Junior partner Member state) unterstützt wird. Eine Übersicht der im Jahr 2009 vergebenen Twinning-Projekte findet sich unter http://ec.europa.eu/enlargement/ pdf/financial_assistance/institution_building/090825_results_for_the_ficheslaunched_during_2009_en.pdf.

Im Jahr 2001 wurden zudem TwinningLight-Projekte als ein flexibleres Mittel für kürzere Projekte eingeführt. TwinningLight-Projekte dauern maximal sechs Monate, können bei Bedarf aber mit Einschränkungen verlängert werden.
Die Unterstützung der Beitritts- oder auch Nachbarstaaten der EU beim Verwaltungsaufbau erfolgt dabei in einem Partnerschaftsprozess mit dem Empfängerstaat (Beneficiary Country), bei dem ein Berater (Resident Twinning Advisor) aus der Verwaltung des jeweiligen Mitgliedsstaates dauerhaft vor Ort ist (dieser entfällt bei Twinning-Light-Projekten) und von Kurzzeitexperten (Short Term Experts) unterstützt wird. Grundlage ihrer Tätigkeit sind dabei die im Proposal angebotenen und mit der Europäischen Kommission vertraglich vereinbarten Projektinhalte, die auch verbindliche Zielstellungen enthalten. Jedes Twinning-Projekt wird von einem Projektleiter aus der projektführenden Verwaltung geleitet. Dieser steuert das Projekt aus dem jeweiligen EUMitgliedsstaat.

Der Sächsische Datenschutzbeauftragte hat gegen Konkurrenz aus anderen EUStaaten für die Bundesrepublik Deutschland den Zuschlag der Europäischen Kommission für ein Twinning-Light-Projekt zur Unterstützung der Datenschutzbehörde in Bosnien-Herzegowina erhalten.

Die Europäische Kommission hat in ihrem aktuellen jährlichen Strategiepapier zur Erweiterung der EU (IP/09/1519 vom 14.10.2009) auf die Fortschritte der westlichen Balkanstaaten auf dem Weg zur europäischen Integration hingewiesen, die im vergangenen Jahr trotz der Schwierigkeiten aufgrund der weltweiten Wirtschaftskrise erzielt wurden. Es werden jedoch auch die Herausforderungen genannt, die die Staaten in den nächsten Monaten und Jahren noch bewältigen müssen. Insbesondere Bosnien-Herzegowina muss demnach dringend die wichtigsten Reformen vorantreiben.

In diesem Projekt wird das bosnischherzegowinische Datenschutzrecht auf Übereinstimmung mit den Vorgaben des EU-Rechts geprüft und auf Anpassungsbedarf hingewiesen. Darüber hinaus sol- len die technischen, juristischen und organisatorischen Fähigkeiten der dortigen Datenschutzbehörde gestärkt werden. Zu diesem Zweck wird es für die Datenschützer des mittelfristig in die EU strebenden Staates Mitarbeiterschulungen und auch einen kurzzeitigen Studienaufenthalt in Deutschland geben. Das Projekt sieht außerdem für ausgewählte bosnisch-herzegowinische Fachbehörden die Erstellung von Handlungshilfen und Schulungen durch den Sächsischen Datenschutzbeauftragten vor.

Während der Projektlaufzeit wird ein Mitarbeiter des Sächsischen Datenschutzbeauftragten als Mid Term Expert dauerhaft in Sarajewo sein und von erfahrenen Experten aus den Verwaltungen des Bundes, Berlins, Schleswig-Holsteins, Hamburgs und Sachsens unterstützt werden.

Die Grundlagen für die Gewährleistung des Grundrechts auf informationelle Selbstbestimmung bestehen in BosnienHerzegowina bereits. Ein Datenschutzgesetz, das sich an der EG-Datenschutzrichtlinie 95/46/EG orientiert, wurde 2006 verabschiedet; eine Datenschutzbehörde wurde 2008 eingerichtet.

Der Sächsische Datenschutzbeauftragte wird bei der Projektdurchführung durch das Bundesverwaltungsamt sowie die Deutsche Gesellschaft für Technische Zusammenarbeit (GTZ) GmbH unterstützt.

Ziel des Projekts ist die Stärkung der dortigen Datenschutzbehörde als eine der wesentlichen Voraussetzungen für die weitere Annäherung Bosnien-Herzegowinas auf seinem Weg in die Europäische Union als Raum der Freiheit, der Sicherheit und des Rechts.

\section{Dr. Tino Naumann \\ Projektleiter}

Der Sächsische Datenschutzbeauftragte

Leiter der Geschäftsstelle

Referent Personalwesen, Kommunales, Steuerrecht, Gesundheitswesen 